\title{
Bifurcation and Feedback Control of an Exploited Prey-Predator System
}

\author{
Uttam Das \\ Department of Mathematics, Sree Chaitanya College, Prafullanagar, North 24 Parganas, Habra, West Bengal 743268, India \\ Correspondence should be addressed to Uttam Das; uttam_das76@yahoo.in
}

Received 22 January 2014; Revised 25 March 2014; Accepted 30 March 2014; Published 22 April 2014

Academic Editor: Min W. Lee

Copyright (C) 2014 Uttam Das. This is an open access article distributed under the Creative Commons Attribution License, which permits unrestricted use, distribution, and reproduction in any medium, provided the original work is properly cited.

\begin{abstract}
This paper makes an attempt to highlight a differential algebraic model in order to investigate the dynamical behavior of a preypredator system due to the variation of economic interest of harvesting. In this regard, it is observed that the model exhibits a singularity induced bifurcation when economic profit is zero. For the purpose of stabilizing the proposed model at the positive equilibrium, a state feedback controller is therefore designed. Finally, some numerical simulations are carried out to show the consistency with theoretical analysis and to illustrate the effectiveness of the proposed controller.
\end{abstract}

\section{Introduction and Model Description}

Biological resources in the prey-predator ecosystem are commercially harvested and sold with the aim of achieving economic interest. For this reason, harvesting plays an important role in the study of biological resources. Furthermore, the harvest effort is usually influenced by the variation of economic interest of harvesting. To formulate a biological economic system from an economic point of view and to investigate the dynamical behavior of the model, many scientists use differential-algebraic equations. The differential equations investigate the dynamics of prey and predators and the algebraic equation studies the harvest effort on prey from an economic perspective.

Differential-algebraic system has been applied widely in power system, aerospace engineering, chemical process, social economic systems, biological systems, network analysis, and so on. With the help of the differential algebraic model for power systems and bifurcation theory, the complex dynamical behavior of power systems, specially the bifurcation phenomena which can reveal instability mechanisms of power systems, has been extensively studied by Marszalek and Trzaska [1], Ayasun et al. [2], Yue and Schlueter [3], and others. Again, the application of differential algebraic model has an immense impact on the analysis of biological system.
I am aware that harvesting has a strong impact on the dynamics of populations. Depending on the applied harvesting strategy, the long run stationary density of a population may be significantly smaller than the long run stationary density of a population without harvesting. Harvesting can lead to the incorporation of a positive extinction probability, even if in the absence of harvesting, a population can be free from extinction risk. If a population is subjected to a positive extinction rate, then harvesting can drive the population density to a dangerously low level at which extinction becomes sure no matter how the harvest affects the population afterwards. Some works on predator-prey species with harvesting can be found in Das et al. [4], Kar et al. [5], Clark [6], and Song and Chen [7]. Kar and Pahari [8], Kar and Ghosh [9], and Kumar et al. [10] have studied the dynamics in prey-predator models with harvesting and have obtained complex dynamics behavior, such as stability of equilibria, Hopf bifurcation, limit cycle, and heteroclinic bifurcation. Zhang and Q.-L. Zhang [11] systematically studied a hybrid predator prey economic model, which is formulated by differential-difference-algebraic equations. They proved that this model exhibits two bifurcations phenomena at the intersampling instants. Kar and Chakraborty [12] have discussed a bioeconomic model with harvesting and removed the singularity induced bifurcation as well as the instability 
behavior towards the positive economic profit by means of feedback control theory.

However, in this context of the above studies, this paper tries to examine the dynamical behavior of a biological economic prey predator model where the prey population is harvested using differential algebraic equation and bifurcation theory. I consider the model with zero economic profit, and singularity induced bifurcation is obtained at the interior equilibrium of the model. To reduce the singularity induced bifurcation, a state feedback controller is designed.

In this paper, I develop a two-species predator-prey model. I consider a logistic growth function of the prey and a Holling type III response function. Thus the growth rate of the prey is formulated as

$$
\frac{d x}{d t}=r x\left(1-\frac{x}{k}\right)-\frac{\alpha x^{2} y}{a+x^{2}}
$$

where $x$ and $y$ represent the prey and predator populations, respectively, at time $t, k$ is the carrying capacity of the prey, $r$ is the intrinsic growth rate of the prey, $\alpha$ is the predation coefficient, and $a$ is the half saturation constant.

The growth rate of the predator population is taken in the following form:

$$
\frac{d y}{d t}=-d y+\frac{\alpha \beta x^{2} y}{a+x^{2}}-\gamma y^{2},
$$

where $\beta$ is the conversion factor (I assume that $\beta<1$, since the whole biomass of the prey is not transferred to the biomass of the predator), $d$ is the death rate of predator, and $\gamma$ is the coefficient of intraspecific competition of the predator population. Here $r, \alpha, \beta, k, d, a, \gamma$ are positive constants.

For considering the exploited prey system, I introduce a scaled harvesting effort $E$ for the prey and then the equations governing my model become

$$
\begin{gathered}
\frac{d x}{d t}=r x\left(1-\frac{x}{k}\right)-\frac{\alpha x^{2} y}{a+x^{2}}-q E x, \\
\frac{d y}{d t}=-d y+\frac{\alpha \beta x^{2} y}{a+x^{2}}-\gamma y^{2} .
\end{gathered}
$$

For system (3), the supply amount of harvested prey into the market is $q E x$ [6], where $q$ is the catchability coefficient of the prey population. Simultaneously, an algebraic equation is also developed by considering the economic interest of harvesting according to Gordon's economic theory of a common property resource [13]. He established the economic interest of the yield of harvest effort as net economic revenue is equal to total revenue (TR) - total cost (TC). In my problem, total revenue (TR) and total cost (TC) in system (3) are given by $\mathrm{TR}=p q E x$ and $\mathrm{TC}=c E$.

Since the net economic revenue $(v)=\mathrm{TR}-\mathrm{TC}$; therefore,

$$
v=(p q x-c) E
$$

where $p$ is the price per unit harvested biomass, $c$ is the cost per unit harvest effort, and $v$ is the economics interest of harvesting.
Based on (3) and (4), a differential algebraic system consisting of two differential equations and one algebraic equation is established as

$$
\begin{gathered}
\frac{d x}{d t}=r x\left(1-\frac{x}{k}\right)-\frac{\alpha x^{2} y}{a+x^{2}}-q E x, \\
\frac{d y}{d t}=-d y+\frac{\alpha \beta x^{2} y}{a+x^{2}}-\gamma y^{2}, \\
0=(p q x-c) E-v .
\end{gathered}
$$

The advantage of the differential algebraic system is that it offers a simpler (than ordinary differential systems) way to study the dynamical behaviour of the system due to the variation of economic interest of harvesting.

The differential-algebraic model (5) can be expressed in the following form:

$$
A \dot{H}(t)=G(x(t), y(t), E(t)),
$$

where

$$
\begin{gathered}
H(t)=(x(t), y(t), E(t))^{T}, \quad A=\left[\begin{array}{lll}
1 & 0 & 0 \\
0 & 1 & 0 \\
0 & 0 & 0
\end{array}\right], \\
G(x(t), y(t), E(t))=\left[\begin{array}{c}
r x\left(1-\frac{x}{k}\right)-\frac{\alpha x^{2} y}{a+x^{2}}-q E x \\
-d y+\frac{\alpha \beta x^{2} y}{a+x^{2}}-\gamma y^{2} \\
(p q x-c) E-v
\end{array}\right] .
\end{gathered}
$$

\section{The Model with Zero Economic Profit}

When the economic profit is zero, system (5) takes the following form:

$$
\begin{gathered}
\frac{d x}{d t}=r x\left(1-\frac{x}{k}\right)-\frac{\alpha x^{2} y}{a+x^{2}}-q E x, \\
\frac{d y}{d t}=-d y+\frac{\alpha \beta x^{2} y}{a+x^{2}}-\gamma y^{2}, \\
0=(p q x-c) E .
\end{gathered}
$$

2.1. The Interior Equilibrium: Existence. The interior equilibrium $P^{*}\left(x^{*}, y^{*}, E^{*}\right)$ exists provided that $c^{2} \alpha \beta>d\left(c^{2}+a p^{2} q^{2}\right)$ and

$$
\begin{gathered}
r k p q \gamma\left(c^{2}+a p^{2} q^{2}\right)^{2}+k c d \alpha p^{2} q^{2}\left(c^{2}+a p^{2} q^{2}\right) \\
>c r \gamma\left(c^{2}+a p^{2} q^{2}\right)^{2}+k \alpha^{2} c^{3} p^{2} q^{2} \beta,
\end{gathered}
$$

where

$$
\begin{gathered}
x^{*}=\frac{c}{p q} \\
y^{*}=\frac{1}{\gamma}\left(-d+\frac{c^{2} \alpha \beta}{c^{2}+a p^{2} q^{2}}\right),
\end{gathered}
$$




$$
\begin{aligned}
E^{*}= & \left(r \gamma k p q\left(a p^{2} q^{2}+c^{2}\right)^{2}+k c \alpha d p^{2} q^{2}\left(c^{2}+a p^{2} q^{2}\right)\right. \\
& \left.-c r \gamma\left(a p^{2} q^{2}+c^{2}\right)^{2}-k \alpha^{2} c^{3} p^{2} q^{2} \beta\right) \\
\times & \left(p q^{2} \gamma k\left(a p^{2} q^{2}+c^{2}\right)^{2}\right)^{-1} .
\end{aligned}
$$

The Jacobian matrix for the differential algebraic equations (DAE's) (8) at an arbitrary point is given by

$$
\begin{aligned}
M & =D_{X} f-D_{E} f\left(D_{E} g\right)^{-1} D_{X} g \\
& =\left[\begin{array}{cc}
r-\frac{2 x r}{k}-\frac{2 a \alpha x y}{\left(a+x^{2}\right)^{2}}-q E+\frac{p q^{2} x E}{p q x-c} & -\frac{\alpha x^{2}}{a+x^{2}} \\
\frac{2 a \alpha \beta x y}{\left(a+x^{2}\right)^{2}} & \frac{\alpha \beta x^{2}}{a+x^{2}}-d-2 y \gamma
\end{array}\right] .
\end{aligned}
$$

2.2. Singular Point and Singularity Induced Bifurcation in a Differential Algebraic Equations (DAEs) System. The DAEs system [14] can be put in the following form:

$$
\begin{array}{ll}
\dot{x}=f(x, y, \mu), & f: \mathfrak{R}^{n+m+l} \longrightarrow \mathfrak{R}^{n}, \\
0=g(x, y, \mu), & g: \mathfrak{R}^{n+m+l} \longrightarrow \mathfrak{R}^{m},
\end{array}
$$

where $x \in \widetilde{X} \subset \Re^{n}, y \in \widetilde{Y} \subset \Re^{m}, \mu \in \Lambda \subset \Re^{l}$ with $n, m$, and $l$ which are all positive integers. In this particular section, $x$ is the dynamic state vector whose time evaluation is directly connected by the equation (12a), $y$ is the instantaneous state vector which satisfies the constraint equation (12b), and the parameter set $\mu$ defines a specific system configuration and operating condition.

Then according to Venkatasubramanian et al. [15], denote the set of all equilibria of the DAEs system (12a)-(12b) to be $\mathrm{EQ}$ and the set of all stable equilibria $\mathrm{OP}$ as

$$
\begin{gathered}
\mathrm{EQ}=\{(x, y, \mu) \in \widetilde{X} \times \widetilde{Y} \times \Lambda: f(x, y, \mu)=0, \\
g(x, y, \mu)=0\}, \\
\mathrm{OP}=\left\{(x, y, \mu) \in E Q: \operatorname{Det}\left(D_{y} g\right) \neq 0,\right. \\
\left.\operatorname{Re}\left(\lambda\left(J_{n}\right)\right)<0\right\},
\end{gathered}
$$

where $\lambda\left(J_{n}\right)$ is the set of all eigenvalues corresponding to the Jacobian matrix $J_{n}=D_{x} f-D_{y} f\left(D_{y} g\right)^{-1} D_{x} g$ of the system (12a)-(12b). Also denote the singular surface $S=\{(x, y, \mu) \in$ $\left.\widetilde{X} \times \widetilde{Y} \times \Lambda: g(x, y, \mu)=0, \Delta(x, y, \mu):=\operatorname{Det}\left(D_{y} g\right)=0\right\}$, and corresponding point on $S$ is known as singular point which plays an important role in differential algebraic system. In a DAEs system the singularity induced bifurcation (SIB) occurs if equilibrium crosses the singular surface $S$ at bifurcation point. Trajectories cross the singularity in a finite time with an infinite speed and the system may change its stability due to an eigenvalue diverging to infinity. This type of bifurcation can be analyzed with the help of the following theorem.
Theorem 1 (singularity induced bifurcation theorem). Suppose that the system (12a)-(12b) satisfies the following conditions at the singular equilibrium $\left(x_{0}, y_{0}, \mu_{0}\right)$.

SIB1: $D_{y} g$ has a simple zero eigenvalue and trace $\left(D_{y} f\left(\operatorname{adj}\left(D_{y} g\right)\right) D_{x} g\right)$ is nonzero.

$$
\begin{aligned}
& \text { SIB2: }\left(\begin{array}{ll}
D_{x} f & D_{y} f \\
D_{x} g & D_{y} g
\end{array}\right) \text { is nonsingular. } \\
& \text { SIB3: }\left(\begin{array}{lll}
D_{x} f & D_{y} f & D_{\mu} f \\
D_{x} g & D_{y} g & D_{\mu} g \\
D_{x} \Delta & D_{y} \Delta & D_{\mu} \Delta
\end{array}\right) \text { is also nonsingular. }
\end{aligned}
$$

Then according to Venkatasubramanian et al. [15], there exists a smooth curve of the equilibrium in $\Re^{n+m+l}$ which passes through $\left(x_{0}, y_{0}, \mu_{0}\right)$ and is transversal to the singular surface at $\left(x_{0}, y_{0}, \mu_{0}\right)$. When $\mu$ increases through $\mu_{0}$, one eigenvalue of the Jacobian matrix $J_{n}=D_{x} f-$ $D_{y} f\left(D_{y} g\right)^{-1} D_{x} g$ of the system (12a)-(12b) moves from $C^{-}$ to $C^{+}$if $M / N>0$ (resp., from $C^{+}$to $C^{-}$if $M / N<0$ ) along the real axis by diverging through infinity. The rest of the $(n-1)$ eigenvalues remain bounded and stay away from the origin. The constants $M$ and $N$ can be computed by evaluating the following:

$$
\begin{aligned}
& M=-\operatorname{trace}\left(D_{y} f\left(\operatorname{adj}\left(D_{y} g\right)\right) D_{x} g\right), \\
& N=D_{\mu} \Delta-\left(\begin{array}{ll}
D_{x} \Delta & D_{y} \Delta
\end{array}\right)\left(\begin{array}{ll}
D_{x} f & D_{y} f \\
D_{x} g & D_{y} g
\end{array}\right)^{-1}\left(\begin{array}{c}
D_{\mu} f \\
D_{\mu} g
\end{array}\right) .
\end{aligned}
$$

Theorem 2. The differential-algebraic model (5) has a singularity induced bifurcation at the interior equilibrium $P^{*}$, and the economic parameter $v=0$ ( $v$ is the economic interest of harvesting) is a bifurcation value. Furthermore, a stability switch occurs as $v$ increases through 0 .

Proof. Let

$$
\begin{gathered}
f(X, E, v)=\left[\begin{array}{c}
r x\left(1-\frac{x}{k}\right)-\frac{\alpha x^{2} y}{a+x^{2}}-q E x \\
-d y+\frac{\alpha \beta x^{2} y}{a+x^{2}}-\gamma y^{2}
\end{array}\right], \\
g(X, E, v)=E(p q x-c)-v,
\end{gathered}
$$

where $X=(x, y)^{T}$ and $v$ is a bifurcation parameter.

It can be calculated that

$$
\left.\operatorname{trace}\left(D_{E} f \operatorname{adj}\left(D_{E} g\right) D_{X} g\right)\right|_{P^{*}}=-q c E^{*} \neq 0 .
$$

Furthermore, it can also be calculated that

$$
\left|\begin{array}{ll}
D_{X} f & D_{E} f \\
D_{X} g & D_{E} g
\end{array}\right|_{P^{*}}=-p q^{2} \gamma x^{*} y^{*} E^{*} \neq 0 .
$$

According to the part A of Venkatasubramanian et al. [15], $\Delta(X, E, v)$ can be defined as follows:

$$
\Delta(X, E, v)=\operatorname{det}\left(D_{E} g\right)=p q x-c .
$$

Now,

$$
\left|\begin{array}{lll}
D_{X} f & D_{E} f & D_{v} f \\
D_{X} g & D_{E} g & D_{v} g \\
D_{X} \Delta & D_{E} \Delta & D_{v} \Delta
\end{array}\right|_{P^{*}}=-p q^{2} \gamma x^{*} y^{*} \neq 0 .
$$


Based on the above analysis, three items can be obtained as follows.

(i) $\left.D_{E} g(X, E, v)\right|_{P^{*}}$ has an algebraically simple zero eigenvalue and trace $\left.\left(D_{E} f \operatorname{adj}\left(D_{E} g\right) D_{X} g\right)\right|_{P^{*}} \neq 0$.

(ii)

$$
\left[\begin{array}{ll}
D_{X} f & D_{E} f \\
D_{X} g & D_{E} g
\end{array}\right]
$$

is nonsingular at $P^{*}$.

(iii) It can be shown that

$$
\left[\begin{array}{lll}
D_{X} f & D_{E} f & D_{v} f \\
D_{X} g & D_{E} \mathcal{g} & D_{v} g \\
D_{X} \Delta & D_{E} \Delta & D_{v} \Delta
\end{array}\right]
$$

is nonsingular at $P^{*}$.

It is observed from (i) to (iii) that all the conditions for singularity induced bifurcation (Venkatasubramanian et al. [15]) are satisfied. Hence the differential-algebraic model system (5) has a singularity induced bifurcation at the positive equilibrium $P^{*}$ and the bifurcation value is $v=0$. Again it is noted that

$$
\begin{aligned}
& M_{1}=-\left.\operatorname{trace}\left(D_{E} f \operatorname{adj}\left(D_{E} g\right) D_{X} g\right)\right|_{P^{*}}=q c E^{*}
\end{aligned}
$$

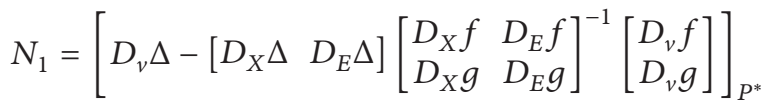

$$
\begin{aligned}
& =\frac{1}{E^{*}} \\
& \frac{M_{1}}{N_{1}}=q c\left(E^{*}\right)^{2}>0 .
\end{aligned}
$$

The inequality (23) satisfies Theorem 3 in Venkatasubramanian et al. [15]. According to Theorem 3 [15] when $v$ increases through 0 , one eigenvalue (denoted by $\lambda_{1}$ ) of the differential-algebraic model (5) moves from $C^{-}$to $C^{+}$along the real axis by diverging through infinity. Consequently, the movement behavior of this eigenvalue influences the stability of the differential-algebraic model (5).

Other eigenvalues of the differential-algebraic model (5) at $P^{*}$ can be calculated as follows. The Jacobian of the differential-algebraic model (5) evaluated at $P^{*}$ takes the following form:

$$
J_{P^{*}}=\left[\begin{array}{ccc}
r-\frac{2 x^{*} r}{k}-\frac{2 a \alpha x^{*} y^{*}}{\left(a+\left(x^{*}\right)^{2}\right)^{2}}-q E^{*} & -\frac{\alpha\left(x^{*}\right)^{2}}{a+\left(x^{*}\right)^{2}} & -q x^{*} \\
\frac{2 a \alpha \beta x^{*} y^{*}}{\left(a+\left(x^{*}\right)^{2}\right)^{2}} & -\gamma y^{*} & 0 \\
p q E^{*} & 0 & 0
\end{array}\right] \text {. }
$$

TABLE 1: Signs of real parts of eigenvalues of model (5) at $P^{*}$.

\begin{tabular}{lcc}
\hline & $\operatorname{Re} \lambda_{1}$ & $\operatorname{Re} \lambda_{2}$ \\
\hline$v<0$ & - & - \\
$v>0$ & + & - \\
\hline
\end{tabular}

According to the leading matrix $A$ in the model (6) and $J_{P^{*}}$, I obtain the characteristic equation of the differentialalgebraic model (5) at $P^{*}$ as $\operatorname{det}\left(\lambda A-J_{P^{*}}\right)=0$, which can be expressed as follows: $\lambda+\gamma y^{*}=0$. Consequently, it can calculate that another eigenvalue of the differentialalgebraic model (5) at $P^{*}$ is $\lambda_{2}=-\gamma y^{*}$. It shows that the eigenvalue $\lambda_{2}$ is negative. And hence the eigenvalue $\lambda_{2}$ is continuous, nonzero, and cannot jump from one half-open complex plane to another as $v$ increases through 0 . Therefore they are continuous and bounded in the $C^{-}$half plane as $v$ increases through 0 and their movement behaviors has no influence on the stability of the differential-algebraic model (5) at the positive equilibrium $P^{*}$.

Table 1 shows the change in the sings of the real parts of the eigenvalues $\lambda_{1}$ and $\lambda_{2}$ due to variation of economics interest of harvest effort.

According to Table 1 and the stability theory, it can be concluded that the differential-algebraic model (5) is stable at $P^{*}$ as $v<0$ and it is unstable at $P^{*}$ as $v>0$. Consequently, a stability switch occurs as $v$ increases through 0 . This completes the proof.

\section{State Feedback Control for Singularity Induced Bifurcation}

In consequence to the above theorem, it is clear that the differential algebraic model (5) becomes unstable when the economic interest of harvesting is considered to be positive. A state feedback controller is designed to eliminate the singularity induced bifurcation and stabilize the differentialalgebraic model (5) at the interior equilibrium $P^{*}$ of the model (8).

According to the leading matrix

$$
A=\left[\begin{array}{lll}
1 & 0 & 0 \\
0 & 1 & 0 \\
0 & 0 & 0
\end{array}\right]
$$

in the model and $J_{P^{*}}$ in (24), it can be calculated that rank $\left(J_{P^{*}}, A J_{P^{*}}, A^{2} J_{P^{*}}\right)=3$. By using Theorem 2-2.1 in Dai [16], it is shown that the differential-algebraic model (5) is locally controllable at $P^{*}$. Consequently, a state feedback controller can be applied to stabilize the differential-algebraic model (5) at the interior equilibrium $P^{*}$ of the model (5).

By using the Theorem 3-1.2 in Dai [16], a state feedback controller $u=s\left(E-E^{*}\right)$ ( $s$ is a feedback gain and $E^{*}$ is the component of the interior equilibrium $P^{*}$ ) can be applied to stabilize the differential-algebraic model (5) at the interior equilibrium $P^{*}$. 
Apply the controller $u=s\left(E-E^{*}\right)$ into the differentialalgebraic model (5) and then a controlled differentialalgebraic model is as follows:

$$
\begin{gathered}
\frac{d x}{d t}=r x\left(1-\frac{x}{k}\right)-\frac{\alpha x^{2} y}{a+x^{2}}-q E x \\
\frac{d y}{d t}=-d y+\frac{\alpha \beta x^{2} y}{a+x^{2}}-\gamma y^{2} \\
0=(p q x-c) E+s\left(E-E^{*}\right)-v
\end{gathered}
$$

Theorem 3. If the feedback gain s satisfies the inequality

$$
\begin{gathered}
s>\max \left\{\left(\left(a+\left(x^{*}\right)^{2}\right)^{2} p q^{2} x^{*} E^{*} k\right)\right. \\
\times\left(y^{*} k \gamma\left(a+\left(x^{*}\right)^{2}\right)^{2}+q E^{*} k\left(a+\left(x^{*}\right)^{2}\right)^{2}\right. \\
+2 x^{*} \gamma\left(a+\left(x^{*}\right)^{2}\right)^{2}+2 a k \alpha x^{*} y^{*} \\
\left.-r k\left(a+\left(x^{*}\right)^{2}\right)^{2}\right)^{-1}, \\
\left.\frac{p q^{2} \gamma E^{*}\left(a+\left(x^{*}\right)^{2}\right)^{3}}{2 a \alpha^{2} \beta\left(x^{*}\right)^{2}}\right\},
\end{gathered}
$$

then the differential-algebraic model (26) is stable at $P^{*}$ of the model (8).

Proof. The Jacobian of the differential-algebraic model (26) evaluated at the interior equilibrium $P^{*}$ takes the following form:

$$
\begin{aligned}
& J_{P^{*}}^{\wedge} \\
& =\left[\begin{array}{ccc}
r-\frac{2 x^{*} r}{k}-\frac{2 a \alpha x^{*} y^{*}}{\left(a+\left(x^{*}\right)^{2}\right)^{2}}-q E^{*} & -\frac{\alpha\left(x^{*}\right)^{2}}{a+\left(x^{*}\right)^{2}} & -q x^{*} \\
\frac{2 a \alpha \beta x^{*} y^{*}}{\left(a+\left(x^{*}\right)^{2}\right)^{2}} & -\gamma y^{*} & 0 \\
p q E^{*} & 0 & s
\end{array}\right] .
\end{aligned}
$$

The characteristic equation of the matrix $J_{P^{*}}^{\wedge}$ is given by

$$
\lambda^{\wedge^{2}}+\Gamma_{1} \lambda^{\wedge}+\Gamma_{2}=0
$$

where

$$
\begin{gathered}
\Gamma_{1}=\frac{2 x^{*} r}{k}-r+\gamma y^{*}+q E^{*}+\frac{2 a \alpha x^{*} y^{*}}{\left(a+\left(x^{*}\right)^{2}\right)^{2}}-\frac{p q^{2} x^{*} E^{*}}{s}, \\
\Gamma_{2}=\frac{2 a \alpha^{2} \beta\left(x^{*}\right)^{3} y^{*}}{\left(a+\left(x^{*}\right)^{2}\right)^{3}}-\frac{p q^{2} \gamma x^{*} y^{*} E^{*}}{s} .
\end{gathered}
$$

By using the Routh-Hurwitz criteria (Kot, [17]), it can be concluded that the model (26) is stable at the equilibrium $P^{*}$, of the system (8), if the feedback gain $s$ satisfies the following condition:

$$
\begin{aligned}
& s>\max \left\{\left(a+\left(x^{*}\right)^{2}\right)^{2} p q^{2} x^{*} E^{*} k\right. \\
& \times\left(y^{*} k \gamma\left(a+\left(x^{*}\right)^{2}\right)^{2}+q E^{*} k\left(a+\left(x^{*}\right)^{2}\right)^{2}\right. \\
& +2 x^{*} \gamma\left(a+\left(x^{*}\right)^{2}\right)^{2}+2 a k \alpha x^{*} y^{*} \\
& \left.-r k\left(a+\left(x^{*}\right)^{2}\right)^{2}\right)^{-1} \\
& \left.\frac{p q^{2} \gamma E^{*}\left(a+\left(x^{*}\right)^{2}\right)^{3}}{2 a \alpha^{2} \beta\left(x^{*}\right)^{2}}\right\} \text {. }
\end{aligned}
$$

Therefore, applying the feedback controller into the model (5), the system can be stabilized around the interior equilibrium and the impulsive phenomenon due to singularity induced bifurcation can be eliminated. This elimination of singularity induced bifurcation implies that the ecological balance in the prey-predator system is restored.

\section{Numerical Simulation}

With the help of Matlab 7.0, and Mathematica 5.2, a numerical simulation is provided to substantiate the theoretical result which has been established in the previous sections of this paper. The parameters values are set in appropriate units as follows: $r=1.5, k=10, q=1.1, \beta=0.2, d=0.002, \gamma=0.03$, $p=1.8, c=1.6, a=1.0$, and $\alpha=0.9$. By virtue of these given parameters, the differential-algebraic model (5) takes the following form:

$$
\begin{gathered}
\frac{d x}{d t}=1.5 x\left(1-\frac{x}{10}\right)-\frac{0.9 x^{2} y}{1+x^{2}}-1.1 E x, \\
\frac{d y}{d t}=-0.002 y+\frac{0.18 x^{2} y}{1+x^{2}}-0.03 y^{2}, \\
0=(1.98 x-1.6) E-v .
\end{gathered}
$$

When economic interest is zero (i.e., $v=0$ ), the system (32) has a positive equilibrium $P^{*}(0.808081,2.303557$, $0.332077)$. When $v=-0.0002$, the eigenvalues are -0.0692 , -973.5813 and then they become $-0.0691,967.2611$ when $v=0.0002$. It is obvious that one eigenvalue remains almost constant and another one moves from $C^{-}$to $C^{+}$along the real axis by diverging through $\infty$.

A state feedback controller $u=s(E-0.332077)$ can be applied to stabilize the differential-algebraic model (32) 


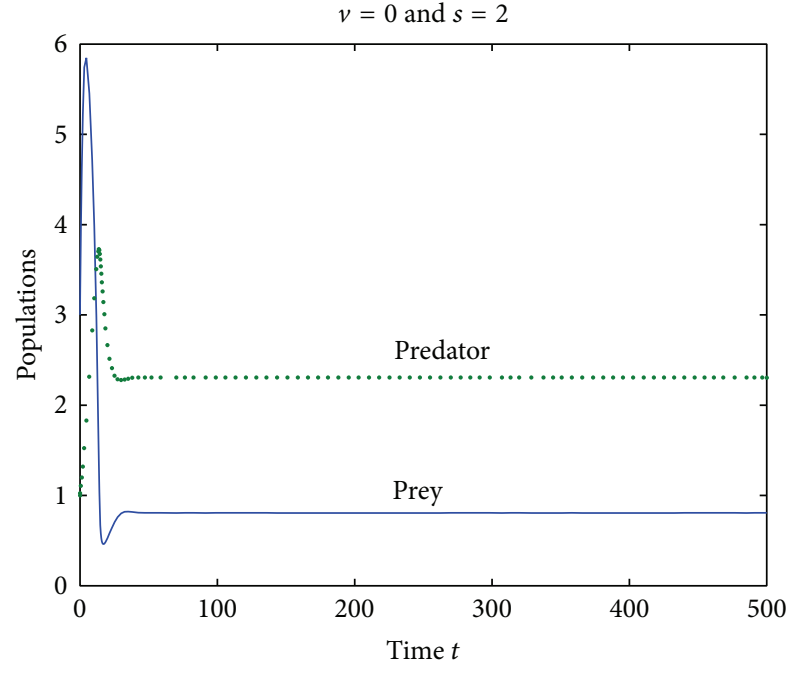

FIGURE 1: Variation of prey and predator populations with the increasing time when $v=0$ and $s=2$.

at $P^{*}$, and then the differential-algebraic model (32) with the state feedback controller takes the following form:

$$
\begin{gathered}
\frac{d x}{d t}=1.5 x\left(1-\frac{x}{10}\right)-\frac{0.9 x^{2} y}{1+x^{2}}-1.1 E x, \\
\frac{d y}{d t}=-0.002 y+\frac{0.18 x^{2} y}{1+x^{2}}-0.03 y^{2}, \\
0=(1.98 x-1.6) E+s(E-0.332077)-v .
\end{gathered}
$$

By using Theorem 3, if the feedback gain $s$ satisfies $s>$ 1.44998 , then the differential-algebraic model (33) is stable at $P^{*}$ and the singularity induced bifurcation of the differentialalgebraic model (32) is also eliminated. Now considering the feedback gain $s=2$, which is greater than 1.44998, I find that an interior equilibrium of the differential algebraic model (33) is $(0.808079,2.303551,0.332081)$, when $v=0$, and the eigenvalues are $-0.0691,-169298.8247$, and consequently the system is stable (see Figure 1). Also when $s=2$ and $v=$ 0.0002 , an interior equilibrium of the differential algebraic model (33) becomes $(0.808011,2.303309,0.332203)$ and the eigenvalues are $-0.0691,-4222.0930$, and consequently the system is stable (see Figure 2) in this case also.

\section{Concluding Remarks}

In this paper, I proposed a harvested differential-algebraic prey-predator model, which is governed by two differential equations and an algebraic equation. The differential equations investigate the dynamics of prey and predator and the algebraic equation studies the harvest effort on prey from an economic perspective. Dynamical behavior of the model is investigated due to the variation of the economic interest of harvesting. It is found that singularity induced bifurcation takes place when the net economic revenue is zero. In consequence to the aforesaid bifurcation, an impulsive phenomenon occurs and the system becomes unstable

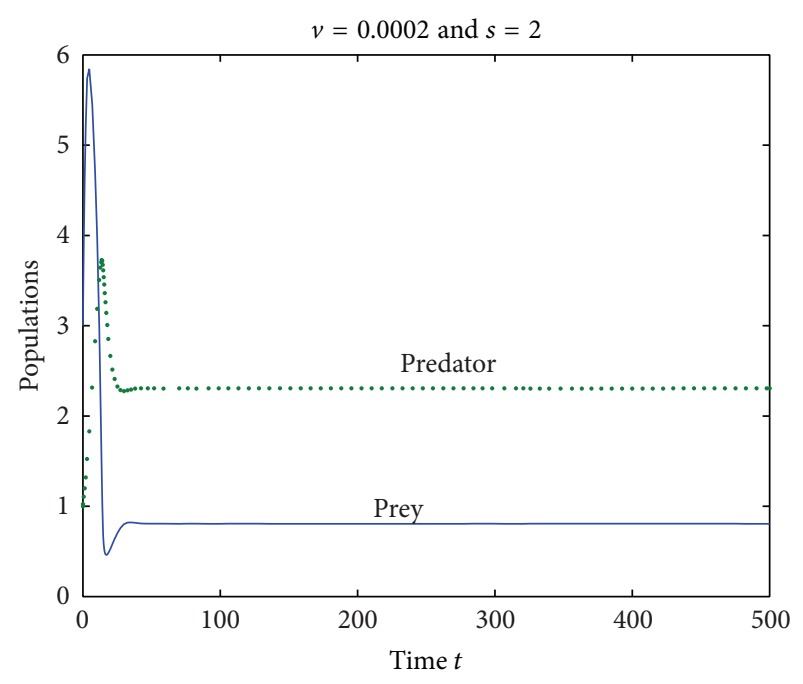

FIGURE 2: Variation of prey and predator populations with the increasing time when $v=0.0002$ and $s=2$.

around the interior equilibrium. A state feedback controller is designed to eliminate the singularity induced bifurcation and stabilize the differential-algebraic model around the interior equilibrium. Numerical simulation is used to show the effectiveness of the state feedback controller. The biological meaning of the proposed controller is that enhancing the harvest effort on preys can not only prevent the stability switch of the prey-predator model, but also drive the model to a stable equilibrium.

However the model and its dynamical behavior are studied mainly on the deterministic framework. In this regard, I can say that it will be more realistic to consider the model in a stochastic environment due to either ecological or economic fluctuations. This needs further future work in this context.

\section{Conflict of Interests}

The author declares that there is no conflict of interests regarding the publication of this paper.

\section{Acknowledgment}

The author is very thankful to his supervisor Dr. Tapan Kumar Kar, Department of Mathematics, Bengal Engineering and Science University, Shibpur, for his valuable comments and suggestions in the preparation of this paper.

\section{References}

[1] W. Marszalek and Z. W. Trzaska, "Singularity-induced bifurcations in electrical power systems," IEEE Transactions on Power Systems, vol. 20, no. 1, pp. 312-320, 2005.

[2] S. Ayasun, C. O. Nwankpa, and H. G. Kwatny, "Computation of singular and singularity induced bifurcation points of differential-algebraic power system model," IEEE Transactions on Circuits and Systems I: Regular Papers, vol. 51, no. 8, pp. 15251538, 2004. 
[3] M. Yue and R. Schlueter, "Bifurcation subsystem and its application in power system analysis," IEEE Transactions on Power Systems, vol. 19, no. 4, pp. 1885-1893, 2004.

[4] U. Das, T. K. Kar, and U. K. Pahari, "Global Dynamics of an exploited prey-predator model with constant prey refuge," ISRN Biomathematics, vol. 2013, Article ID 637640, 12 pages, 2013.

[5] T. K. Kar, K. Chakraborty, and U. K. Pahari, "A prey-predator model with alternative prey mathematical model and analysis," Canadian Applied Mathematics Quarterly, vol. 18, no. 2, pp. 137$167,2010$.

[6] C. W. Clark, Mathematical Bioeconomics: The Optimal Management of Renewable Resource, John Wiley and Sons, New York, NY, USA, 2nd edition, 1990.

[7] X. Song and L. Chen, "Optimal harvesting and stability for a two-species competitive system with stage structure," Mathematical Biosciences, vol. 170, no. 2, pp. 173-186, 2001.

[8] T. K. Kar and U. K. Pahari, "Modelling and analysis of a preypredator system with stage-structure and harvesting," Nonlinear Analysis: Real World Applications, vol. 8, no. 2, pp. 601-609, 2007.

[9] T. K. Kar and B. Ghosh, "Bifurcations and feedback control of a stage-structure exploited prey-predator system," International Journal of Environmental Science and Technology, vol. 2, no. 6, pp. 131-141, 2010.

[10] S. Kumar, S. K. Srivastava, and P. Chingakham, "Hopf bifurcation and stability analysis in a harvested one-predator-two-prey model," Applied Mathematics and Computation, vol. 129, no. 1, pp. 107-118, 2002.

[11] X. Zhang and Q.-L. Zhang, "Bifurcation analysis and control of a class of hybrid biological economic models," Nonlinear Analysis: Hybrid Systems, vol. 3, no. 4, pp. 578-587, 2009.

[12] T. K. Kar and K. Chakraborty, "Bioeconomic modeling of a prey predator system using differential algebraic equations," International Journal of Environmental Science and Technology, vol. 2, no. 1, pp. 13-34, 2010.

[13] H. S. Gordon, "The economic theory of a common property resource: the fishery," Journal of Political Economy, vol. 62, pp. 124-142, 1954.

[14] K. E. Brenan, S. L. Campbell, and I. R. Petzold, Numerical Solution of Initial Value Problems in Differential-Algebraic Equations, North Holland, Amsterdam, The Netherlands, 1989.

[15] V. Venkatasubramanian, H. Schattler, and J. Zaborszky, "Local bifurcations and feasibility regions in differential-algebraic systems," IEEE Transactions on Automatic Control, vol. 40, no. 12, pp. 1992-2013, 1995.

[16] L. Dai, Singular Control System, Springer, New York, NY, USA, 1989.

[17] M. Kot, Elements of Mathematical Biology, Cambridge University Press, Cambridge, UK, 2001. 


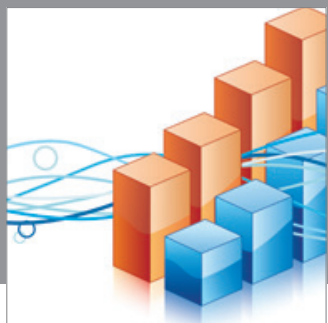

Advances in

Operations Research

mansans

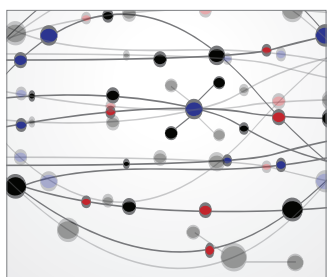

The Scientific World Journal
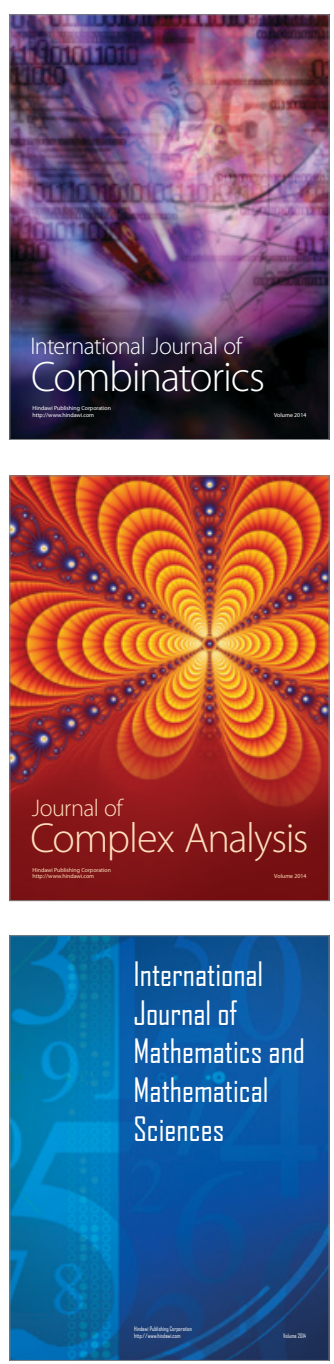
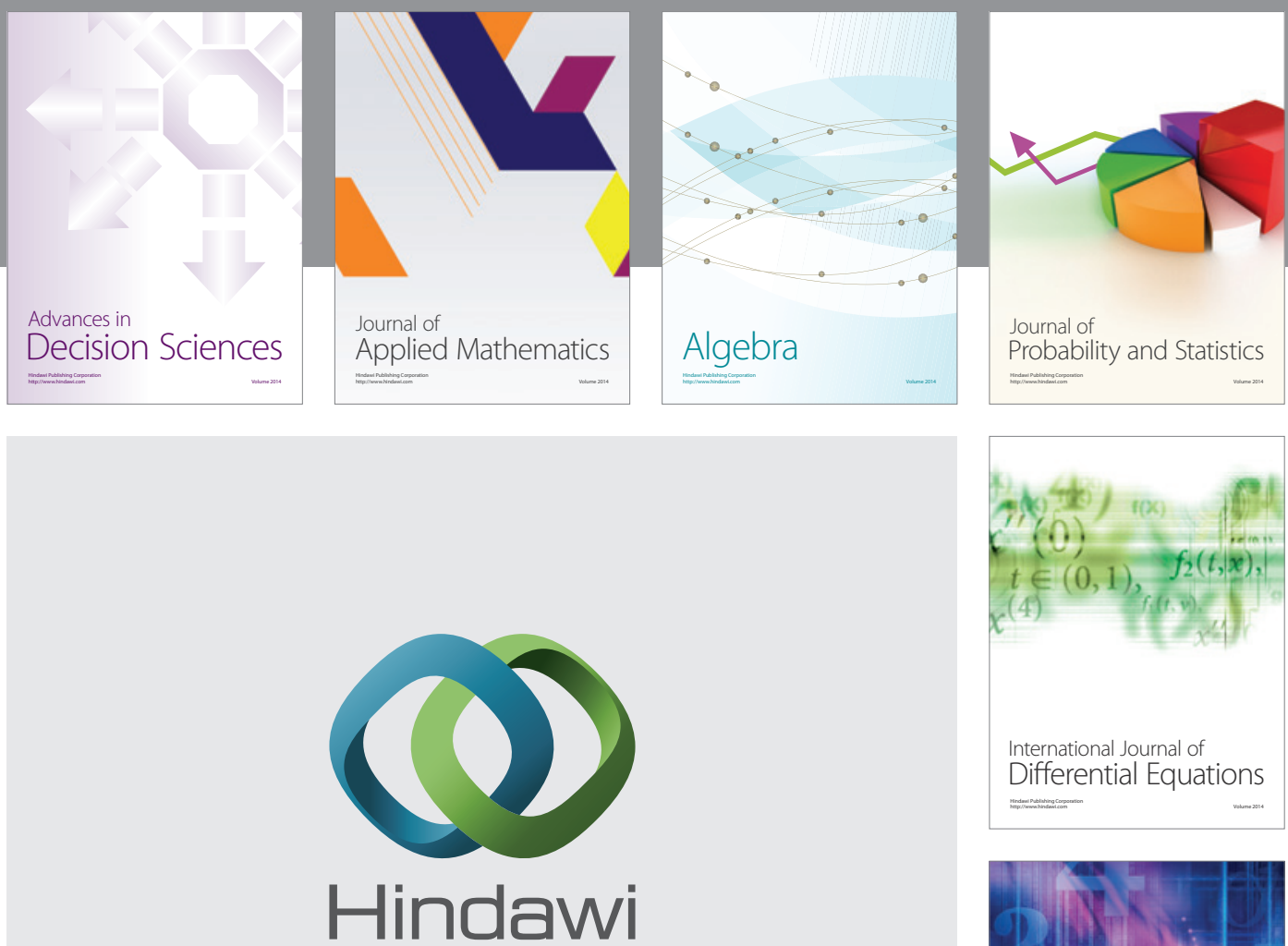

Submit your manuscripts at http://www.hindawi.com
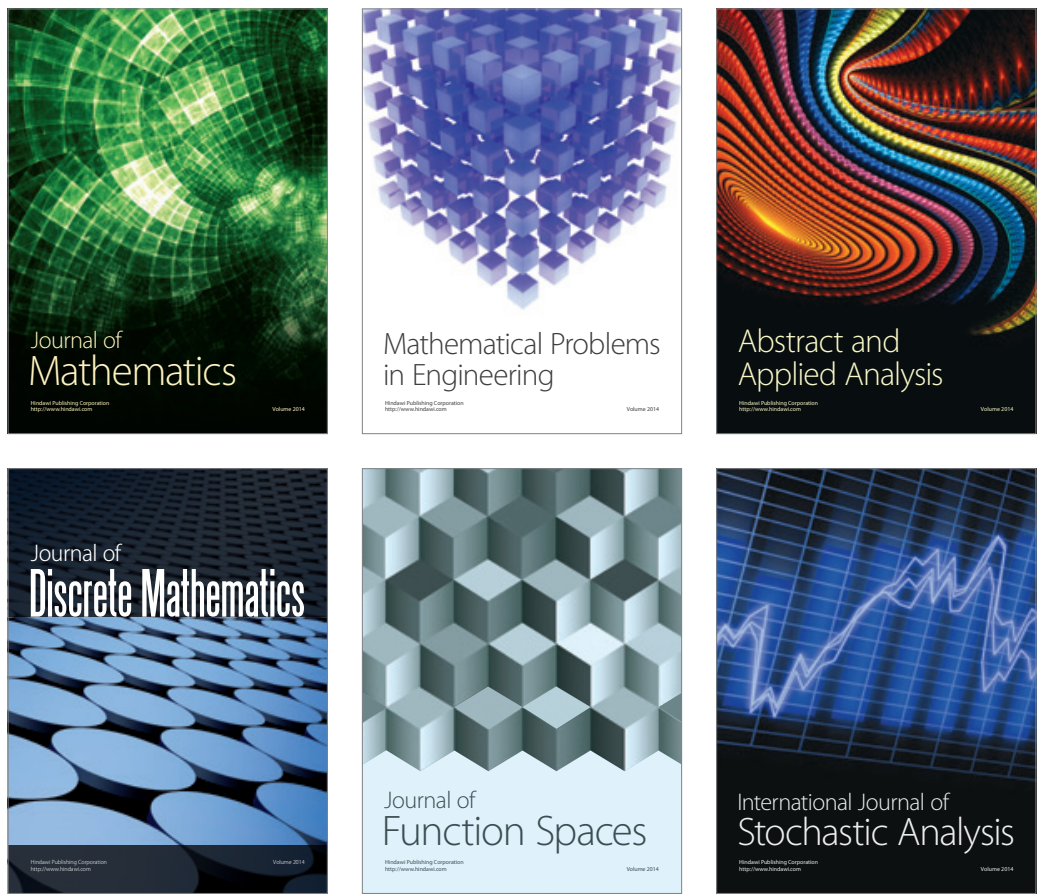

Journal of

Function Spaces

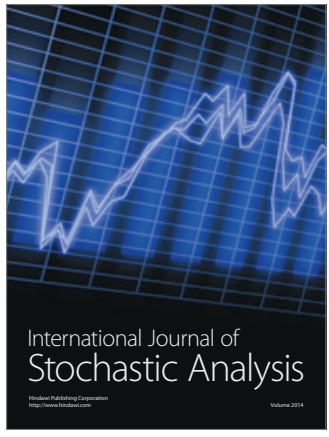

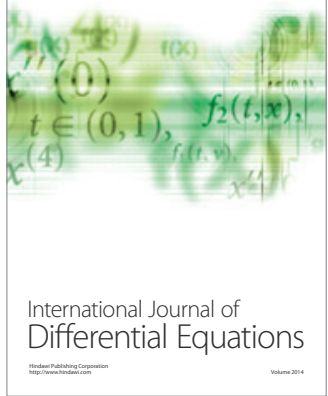
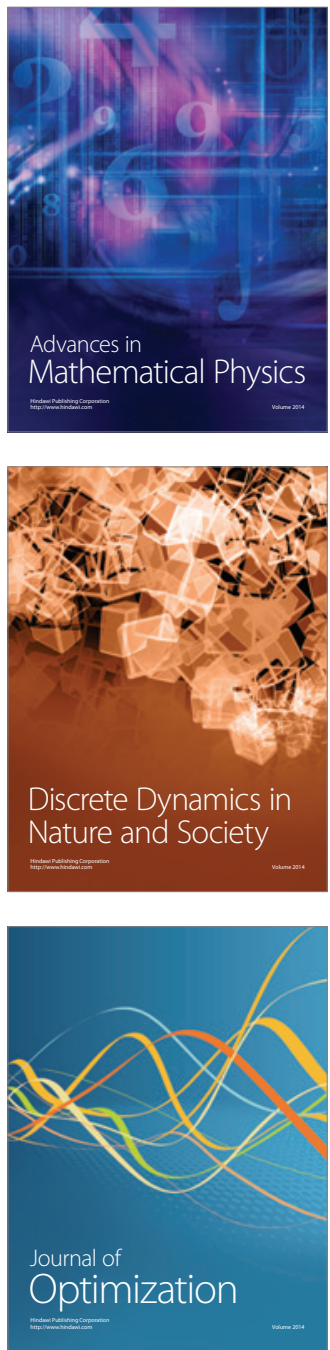